\title{
A técnica de fotoluminescência aplicada à investigação de imperfeições estruturais em poços quânticos de materiais semicondutores
}

\section{The photoluminescence technique applied to the investigation of structural imperfections in quantum wells of semiconducting materials}

\author{
Edson Laureto ${ }^{1}$; Ivan Frederico Lupiano Dias²; José Leonil Duarte²; Dari de Oliveira \\ Toginho Filho ${ }^{2}$; Sidney Alves Lourenço ${ }^{3}$; Eliermes Arraes Meneses ${ }^{4}$
}

\begin{abstract}
Resumo
Fotoluminescência é uma das técnicas de espectroscopia mais utilizadas no estudo das propriedades ópticas de materiais e heteroestruturas semicondutoras. Neste trabalho, é explorada a potencialidade desta técnica na investigação e caracterização de imperfeições estruturais decorrentes de flutuações na composição química de ligas ternárias e quaternárias, rugosidades de interface, e compostos estequiometricamente indesejados decorrentes da troca entre elementos químicos nas interfaces. São analisadas amostras de poços quânticos formados por heteroestruturas de GaAs/AlGaAs, GaAsSb/ GaAs, GaAsSbN/GaAs e GaAs/GaInP, tendo como objetivo verificar a influência das imperfeições estruturais sobre os espectros de PL.

Palavras-chave: Semicondutores. Poços quânticos. Interfaces. Fotoluminescência.
\end{abstract}

\begin{abstract}
Photoluminescence is one of the most used spectroscopy techniques for the study of the optical properties of semiconducting materials and heterostructures. In this work the potentiality of this technique is explored through the investigation and characterization of structural imperfections originated from fluctuations in the chemical composition of ternary and quaternary alloys, from interface roughnesses, and from unintentional compounds formed by the chemical elements intermixing at the interfaces. Samples of GaAs/AlGaAs, GaAsSb/GaAs, GaAsSbN/GaAs and GaAs/GaInP quantum well structures are analyzed to verify the influence of the structural imperfections on the PL spectra.
\end{abstract}

Key words: Semiconductors. Quantum wells. Interfaces. Photoluminescence.

\footnotetext{
1 Departamento de Física, Universidade Estadual de Londrina, Londrina-Pr, Brasil. laureto@uel.br(Edson Laureto).

2 Departamento de Física, Universidade Estadual de Londrina, Londrina-Pr, Brasil.

3 Centro de Ciências Exatas e Tecnológicas, Universidade Norte do Paraná, Londrina-Pr, Brasil.

${ }^{4}$ Instituto de Física Gleb Wataghin, Universidade Estadual de Campinas, Campinas-SP, Brasil.
} 


\section{Introdução}

O estudo das propriedades de materiais semicondutores é de grande interesse, inicialmente, do ponto de vista tecnológico, devido a suas aplicações em dispositivos eletrônicos, optoeletrônicos e fotônicos, como transistores, diodos emissores de luz (LED, do inglês Light Emissor Diode), fotodetetores e LASERs (do inglês Light Amplification by Stimulated Emission Radiation) (SHUR, 1990). Além disso, o estudo desses materiais é relevante para a física fundamental, pela possibilidade de estudar o comportamento de portadores de carga (elétrons e buracos) e de complexos formados pela interação entre eles (excitons, biexcitons, trions, etc.) em situações de dimensões quânticas, como, por exemplo, em sistemas de poços, fios e pontos quânticos (CINGOLANI; PLOOG, 1991; YOFFE, 2001; AKIYAMA et al., 2003; XU, 2005); WARE et al., 2005). Este tema ganhou um forte impulso a partir da década de 1970, com o trabalho de Esaki e Tsu (ESAKI; TSU, 1970), no qual se sugeriu a construção de heteroestruturas baseadas em materiais semicondutores com propriedades ópticas e elétricas que poderiam ser moldadas através de "design" específico, e que seriam bastante distintas dos materiais volumétricos.

O aprimoramento dos sistemas de crescimento de cristais artificiais, principalmente daqueles baseados em métodos epitaxiais, como Epitaxia por Feixe Molecular (MBE, do inglês Molecular Beam Epitaxy) e Deposição por Vapor Químico de Organometálicos (MOCVD, do inglês Metal-Organic Chemical Vapor Deposition), possibilitou a confecção de heteroestruturas com alta qualidade cristalina, com um controle muito preciso da composição química dos elementos constituintes dos materiais, e com interfaces muito bem definidas. Isso motivou o surgimento de um grande número de trabalhos científicos, teóricos e experimentais, acerca das propriedades das mais diversas heteroestruturas formadas por materiais e combinações de materiais semicondutores, em especial aqueles formados por elementos das colunas III-A e V-A da Tabela Periódica, conhecidos como semicondutores III-V (SINGH; BAJAJ, 1985; ANDREANI; PASQUARELLO, 1990; VURGAFTMAN; MEYER; RAM- MOHAN, 2001; FERNANDEZ et al., 2003).

Nos estudos experimentais, é utilizada uma vasta gama de técnicas de investigação, em particular as de espectroscopia óptica, dentre as quais uma das mais difundidas é a fotoluminescência (PL, do inglês Photoluminescence). Essa técnica se baseia no processo de emissão radiativa espontânea dos materiais. Pode-se extrair um bom conjunto de informações sobre as propriedades ópticas e estruturais de semicondutores e de heteroestruturas semicondutoras, a partir da análise da dependência de sua emissão espontânea com a energia.

Um número considerável de artigos desta área dedica-se ao estudo de imperfeições estruturais em heteroestruturas, associadas a flutuações localizadas da composição química das ligas, e imperfeições na formação das interfaces (WEISBUCH et al., 1981; ZIMMERMANN; RUNGE, 1997; OLIVEIRA; MENESES; SILVA, 1999; ALESSI et al., 2000; CHEN et al., 2004). Estas imperfeições, relacionadas diretamente com a dinâmica de crescimento do material, afetam sensivelmente os canais de recombinação radiativa destes sistemas, e podem ser investigadas e avaliadas através de medidas de PL. Informações importantes podem ser obtidas adicionalmente variando-se parâmetros relativos à medida, como temperatura, intensidade de excitação, pressão hidrostática, etc.

Neste trabalho, são estudadas as imperfeições estruturais em poços quânticos semicondutores, por meio da técnica de fotoluminescência. Avaliam-se os efeitos das flutuações de composição nas ligas, das rugosidades de interface e de interfaces composicionalmente difusas sobre os espectros ópticos de poços quânticos envolvendo materiais como GaAs/AlGaAs, GaAsSb/AlGaAs, GaAsSbN/ GaAs e GaAs/GaInP. A análise da posição da 
intensidade máxima da emissão, da forma e da largura a meia-altura da curva de PL, confrontada com os parâmetros de crescimento, fornece informações valiosas sobre os aspectos estruturais das amostras investigadas.

O trabalho está dividido da seguinte maneira: na Secção II, serão abordados alguns conceitos físicos básicos acerca de semicondutores e suas heteroestruturas, com ênfase nas propriedades ópticas de poços quânticos. Também estão contidos nessa secção os princípios básicos da técnica de fotoluminescência, além de uma discussão sobre as imperfeições estruturais e de interface e seus efeitos sobre os processos de emissão radiativa nos poços. Na secção III é apresentado um esquema da montagem experimental da técnica de PL, assim como uma descrição das características principais das amostras investigadas neste trabalho. Os resultados experimentais são apresentados e discutidos na Secção IV. Finalizando, a Secção V traz as conclusões oriundas deste trabalho.

\section{Conceitos físicos}

A. Materiais semicondutores, heterojunções e heteroestruturas

As propriedades elétricas e ópticas de um sólido cristalino, tais como a condutividade e a absorção óptica, estão diretamente conectadas com sua estrutura de bandas de energia. As bandas de energia têm origem na sobreposição dos níveis de energia dos átomos, quando estes se agrupam para formar o sólido. Essas bandas têm uma estrutura que está intimamente relacionada com o arranjo cristalino e podem ser consideradas como um mapeamento dos níveis de energia permitidos aos elétrons de valência do sólido com relação ao vetor de onda (k) do elétron. Na Figura 1, é mostrada a estrutura de bandas do semicondutor GaAs (YU; CARDONA, 1996).

A característica principal, do ponto de vista tecnológico, é a lacuna de energia que existe entre o topo da banda de valência (BV) e o fundo da banda de condução (BC), comumente denominada "gap" fundamental. O material será considerado um semicondutor se a energia de seu "gap" fundamental $\left(\mathrm{E}_{\mathrm{g}}\right)$ tiver um valor maior que zero e menor que aproximadamente $3 \mathrm{eV}$. Semicondutores como o Silício e o Germânio possuem "gap" em torno de 1 eV (na região infra-vermelha do espectro eletromagnético), e nitretos como o GaN têm "gap" em torno de $3 \mathrm{eV}$ (região próxima do ultra-violeta). O GaAs tem "gap" de 1,42 $\mathrm{eV}$ em temperatura ambiente.

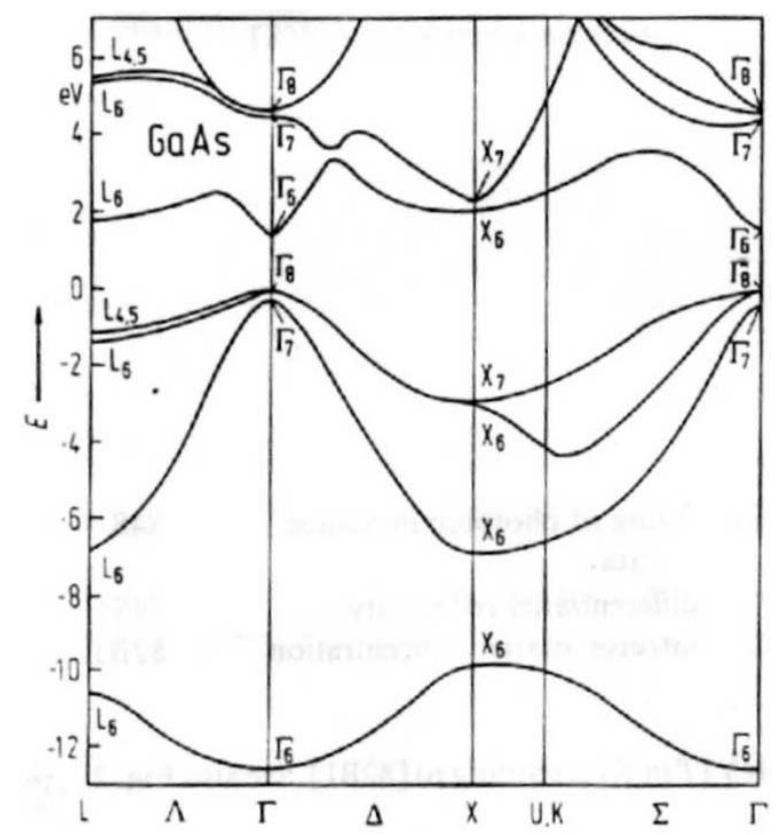

Figura 1. Estrutura de bandas de energia do material GaAs. A referência para o eixo de energia (eixo-y) é o topo da banda de valência, no ponto $\Gamma_{8}$ (YU; CARDONA 1996).

Para aplicações que exigem emissão de luz, como em dispositivos conhecidos como LEDs e LASERs, é interessante que os materiais semicondutores apresentem "gap" direto, ou seja, que o topo de BV e o fundo de BC estejam no mesmo ponto $\mathbf{k}$ da estrutura de bandas. Esse é o caso dos compostos III-V como InP, GaSb e GaAs (veja Figura 1), e das misturas (ou ligas) desses compostos, como $\mathrm{Ga}_{\mathrm{x}} \mathrm{As}_{1 \text { - }}$ $\mathrm{x}$ Sb, $\mathrm{Al}_{\mathrm{x}} \mathrm{Ga}_{1-\mathrm{x}} \mathrm{As}(\mathrm{x}<0,4)$ e $\operatorname{In}_{\mathrm{x}} \mathrm{Ga}_{1-\mathrm{x}} \mathrm{As}_{\mathrm{y}} \mathrm{P}_{1-\mathrm{y}}$, que são a base dos LASERs semicondutores atualmente disponíveis comercialmente. A eficiência na emissão de luz é muito baixa em materiais de "gap" indireto, 
como, por exemplo o Si e o Ge. Os subíndices x e y indicam a composição química de cada elemento constituinte da liga. O "gap" é definido pela composição química, o que possibilita "sintonizar" sua energia em qualquer valor que esteja entre o maior e o menor valor de "gap" dos materiais que constituem a liga. Efeitos de tensão gerados pelo descasamento do parâmetro de rede entre a liga e o substrato sobre o qual ela é depositada alteram o "gap" e interferem nas propriedades ópticas do material, mas não serão considerados neste trabalho. Na tabela I, é dada uma relação de equações que descrevem a variação do "gap" com a composição para algumas ligas tecnologicamente importantes (SINGH, 1993).

Tabela 1. Expressões que descrevem a variação do "gap" fundamental com a composição química de algumas ligas semicondutoras à temperatura ambiente (300 K) (SINGH, 1993).

\begin{tabular}{cc}
\hline Composto & Energia do "gap" (eV) \\
\hline $\mathrm{Al}_{\mathrm{x}} \mathrm{Ga}_{1-\mathrm{x}} \mathrm{As}$ & $1,424+1,455 \mathrm{x}$ \\
$\mathrm{Al}_{\mathrm{x}} \mathrm{In}_{1-\mathrm{x}} \mathrm{As}$ & $0,36+2,012 \mathrm{x}+0,698 \mathrm{x}^{2}$ \\
$\mathrm{Ga}_{\mathrm{x}} \mathrm{In}_{1-\mathrm{x}} \mathrm{P}$ & $1,351+0,643 \mathrm{x}+0,786 \mathrm{x}^{2}$ \\
$\mathrm{Ga}_{\mathrm{x}} \mathrm{In}_{1-\mathrm{x}} \mathrm{As}$ & $0,36+1,064 \mathrm{x}$ \\
$\mathrm{GaAs}_{\mathrm{x}} \mathrm{Sb}_{1-\mathrm{x}}$ & $0,726-0,502 \mathrm{x}+1,2 \mathrm{x}^{2}$ \\
\hline
\end{tabular}

Quando dois semicondutores de "gaps" diferentes são sobrepostos (formando uma heterojunção), a diferença em energia entre os "gaps" divide-se entre a banda de condução $\left(\Delta \mathrm{E}_{\mathrm{c}}\right)$ e a banda de valência $\left(\Delta \mathrm{E}_{\mathrm{v}}\right)$. Gera-se desse modo, uma descontinuidade nos extremos das bandas na interface entre os dois materiais, e um perfil de energia potencial, tanto para elétrons (na BC) quanto para buracos (na BV) que têm a forma de degrau (a altura do degrau na BC (BV) é definida por $\Delta \mathrm{E}_{\mathrm{c}(\mathrm{v})}$ ). Se os materiais são dispostos alternadamente, é possível construir um perfil de potencial tipo barreira ou tipo poço, dependendo do "gap" do material intercalado, formando assim uma heterojunção dupla ou heteroestrutura.

Potenciais do tipo degrau, barreira ou poço influenciam fortemente o movimento dos portadores de carga elétrica, e a análise teórica destes fenômenos foi uma das primeiras aplicações da Mecânica Quântica na década de 1930. Transistores de heterojunção bipolar (HBT, do inglês Heterojunction Bipolar Transistor) e de efeito de campo (FET, do inglês Field Effect Transistor), LASERs e outros dispositivos fotônicos têm seu funcionamento baseado no perfil de energia potencial criado pela heteroestrutura, e na aplicação das leis da Mecânica Quântica para a descrição da dinâmica dos elétrons e buracos (SHUR, 1990).

A Figura 2 mostra um perfil de potencial tipo poço quântico de barreiras finitas. Um perfil deste tipo pode ser formado, por exemplo, pela sobreposição alternada dos materiais $\mathrm{GaAs}\left(\mathrm{E}_{\mathrm{g}}=1,42 \mathrm{eV}\right)$ e $\mathrm{Al}_{0,3} \mathrm{Ga}_{0,7} \mathrm{As}\left(\mathrm{E}_{\mathrm{g}}=1,86 \mathrm{eV}\right)$. Os efeitos quânticos manifestam-se na discretização dos níveis de energia permitidos no interior de poço, decorrência do confinamento dos portadores nesta região. A posição em energia desses níveis pode ser facilmente calculada com base na teoria quântica (BASTARD, 1986), é função sensível da espessura do poço e também dependente da altura da barreira. O nível fundamental para o elétron na banda de condução (buraco na banda de valência) não é mais o fundo (topo) da banda do material que constitui o poço, mas o primeiro nível quântico que surge devido aos efeitos de confinamento. O confinamento renormaliza o "gap" da heteroestrutura. Desse modo, variando a espessura do poço, ou mudando a composição da liga de modo a alterar a altura das barreiras, é possível alterar o "gap" fundamental da heteroestrutura.

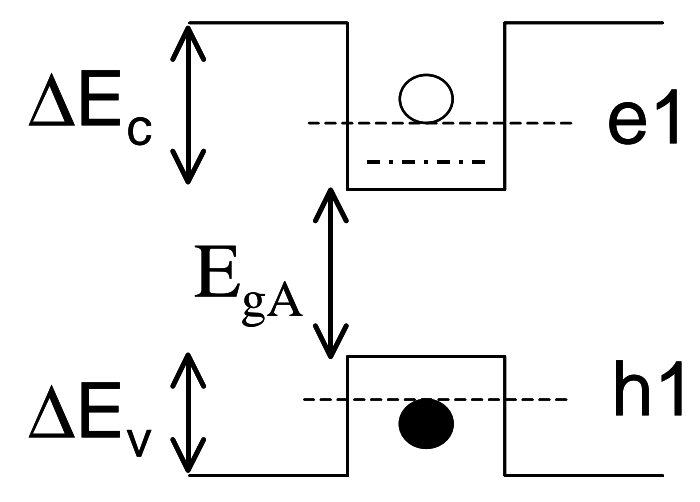

Figura 2. Forma do potencial em uma estrutura tipo poço quântico. 
A altura das barreiras nas bandas de condução e de valência é dada por $\Delta \mathrm{E}_{\mathrm{c}}$ e $\Delta \mathrm{E}_{\mathrm{v}}$, respectivamente. $\mathrm{E}_{\mathrm{gA}}$ é o valor do "gap" do material do poço. As linhas pontilhadas se referem aos níveis quânticos que surgem devido ao confinamento dos portadores. $\mathrm{O}$ círculo vazio representa o elétron em seu nível fundamental na BC, e o círculo cheio representa o buraco em seu nível fundamental na BV. A ligação coulombiana entre o par elétron-buraco dá origem ao exciton, cujo nível é indicado pela linha tracejadapontilhada.

Considerando o eixo z como a direção perpendicular à superfície dos materiais (direção do crescimento), o movimento nas direções x,y não é influenciado pelo confinamento. Desse modo, poços quânticos são sistemas bi-dimensionais. Outros sistemas como fios quânticos (uni-dimensionais) e pontos quânticos (zerodimensionais) também podem ser fabricados a partir de heteroestruturas semicondutoras (DAVIES, 1998), produzidas por técnicas de crescimento epitaxial como MBE e MOCVD.

\section{B. Fotoluminescência}

O processo de emissão espontânea em um semicondutor ocorre em quatro etapas: excitação, relaxação, termalização e recombinação. A excitação é a incidência de luz com energia maior que o "gap" de um semicondutor, que cria pares elétron-buraco mediante a promoção de elétrons de seus estados fundamentais na BV para níveis desocupados na BC. Em seguida, ocorre a relaxação, na qual o excesso de energia adquirido pelos portadores é cedido à rede cristalina por emissão de fônons. Com a termalização que ocorre em seguida, os pares elétron-buraco tendem a ocupar os estados de mais baixa energia possível nos fundos das bandas. Depois de um intervalo de tempo que é em geral extremamente curto (entre $10^{-9}$ e $10^{-12}$ segundos), o elétron retorna para seu nível fundamental recombinando com o buraco, e a recombinação radiativa gera um fóton (luz). É nesse processo geral que se baseia a técnica de fotoluminescência (PL).

A emissão espontânea de poços quânticos semicondutores é produzida mediante o mesmo processo. No entanto, como o nível fundamental para o elétron é o primeiro nível de energia do poço na banda de condução, e para o buraco é o primeiro nível de energia do poço na banda de valência, a recombinação ocorre quando os portadores relaxam para estes níveis de energia fundamentais. O confinamento do elétron e do buraco em uma região espacialmente restringida aumenta a interação entre eles via força de Coulomb, reduzindo ainda mais a energia de emissão (ver Fig. 2). O par elétron-buraco ligado via interação Coulombiana é conhecido como exciton. Estudos experimentais indicam que a emissão espontânea de poços quânticos semicondutores de boa qualidade tem natureza essencialmente excitônica até mesmo a temperatura ambiente (CHRISTEN; BIMBERG, 1990).

\section{Imperfeições de interface e flutuações na composição química das ligas}

As técnicas de crescimento epitaxial permitem um controle muito preciso das interfaces em heteroestruturas. Mas, para obter interfaces de boa qualidade, é necessário um conhecimento bastante profundo do seu processo de formação. Comumente, essas heteroestruturas são constituídas por ligas ternárias e quaternárias, as quais podem apresentar flutuações microscópicas em sua composição química (como será discutido mais adiante). Imperfeições estruturais relacionadas às interfaces e às flutuações de composição sempre existem, em maior ou menor grau, e estão relacionadas com a dinâmica do processo de crescimento. Aqui serão abordados aspectos dessas imperfeições que afetam sensivelmente as propriedades ópticas de poços quânticos de materiais semicondutores. 


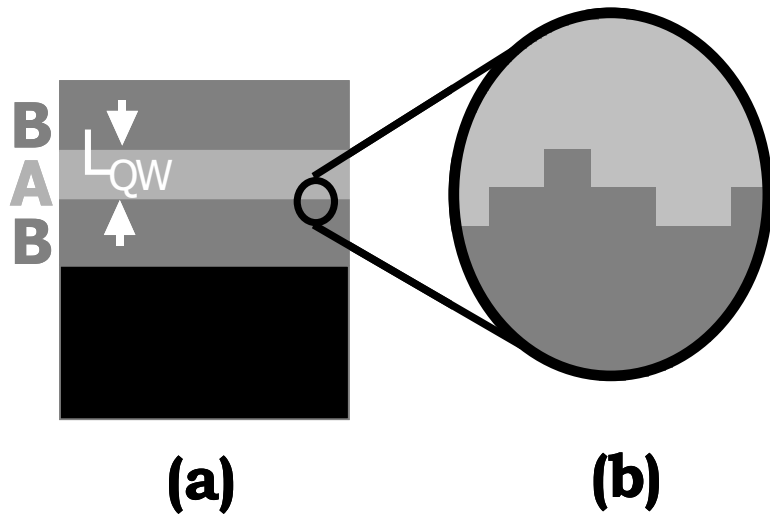

Figura 3. (a) Esquema da estrutura de um poço quântico no espaço real. $\mathrm{L}_{\mathrm{OW}}$ é a largura do poço constituído pelo material A, e as barreiras são constituídas pelo material B. A parte inferior, mais escura, representa o substrato. (b) Representação esquemática da visão microscópica da interface entre os materiais A e B.

A estrutura de um poço quântico no espaço real está representada na Figura 3-(a), onde as interfaces entre os materiais A e B são desenhadas de modo perfeitamente abruptas. Entretanto, uma visão microscópica, como indicado na Figura 3-(b), mostra que as interfaces nunca são perfeitamente abruptas, e contêm rugosidades. Essas imperfeições estruturais são, como ilustrado na Figura 3-(b), ilhas de material $\mathrm{B}$ ocorrendo na região do material A e vales de material A em B.

A interação entre os portadores de carga fotogerados numa medida de PL e as rugosidades de interface pode ser esquematizada considerando uma situação simplificada onde uma das interfaces é perfeitamente abrupta, e a outra possui uma rugosidade de altura igual a uma monocamada atômica (ML). Essa configuração de interfaces está mostrada na Figura 4-(a). Z é a direção do crescimento e o plano x-y é perpendicular a z. Na posição (1) indicada nesta figura, o poço quântico tem espessura igual a $\mathrm{L}_{\mathrm{QW}}$. Logo, os excitons que estão nesta região do poço estão submetidos a um potencial referente ao poço de espessura $\mathrm{L}_{\mathrm{QW}} \cdot \mathrm{Na}$ posição (2), os excitons estão submetidos ao potencial referente ao poço de espessura $\mathrm{L}_{\mathrm{QW}}+1 \mathrm{ML}$, cujos níveis de energia estão deslocados em relação ao

primeiro caso por uma diferença dada por $\Delta \mathrm{E}_{1 \mathrm{ML}}$. Isso está esquematizado na Figura 4-(b).

Imperfeições de outra natureza, mas que levam ao mesmo efeito, estão relacionadas às flutuações de composição química dos materiais. Uma liga semicondutora é caracterizada pela composição química dos elementos que a constituem. Na liga $\mathrm{Al}_{0.3} \mathrm{Ga}_{0.7} \mathrm{As}$, por exemplo, dos sítios da rede cristalina destinados aos elementos do grupo III, 30\% são ocupados por átomos do elemento Alumínio e 70\% por átomos do elemento Gálio, definindo assim a composição global do material. Entretanto, essa distribuição não é perfeitamente homogênea, e variações de composição podem ocorrer de um ponto para outro dentro do material. As flutuações de composição geram variações localizadas na energia do "gap" da liga. Desse modo, tanto a desordem nas interfaces quanto a desordem composicional induzem flutuações no potencial de confinamento do poço quântico.

(2)

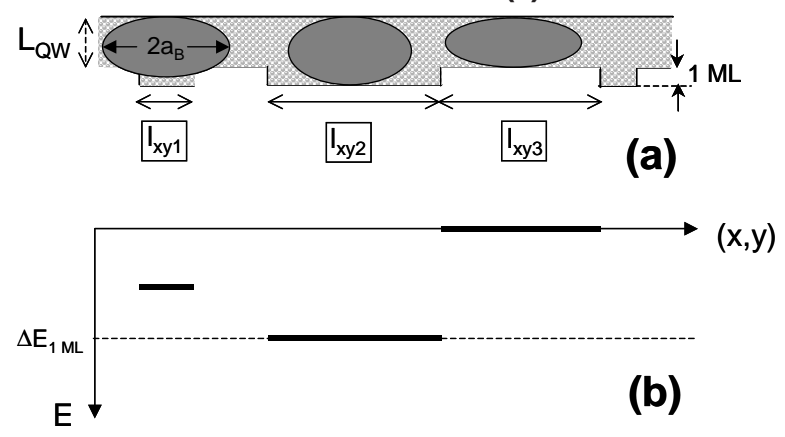

Figura 4. (a) Esquema da estrutura de um poço quântico. A parte hachurada é a região de confinamento. As elipses escuras representam excitons, cujo raio de Bohr é $\mathrm{a}_{\mathrm{B}}$. (b) Posição da energia de excitons confinados em diferentes posições ao longo do plano $(\mathrm{x}, \mathrm{y})$ do poço.

A configuração resultante é a formação de um potencial que depende da posição no plano x-y, como visualizado na Figura 5. Este potencial é aleatoriamente distribuído ao longo desse plano, devido à distribuição aleatória da forma (dimensão lateral e profundidade) das rugosidades de interface. Nos mínimos desse potencial, são criados estados cujas energias são mais baixas que as do estado 
bidimensional do poço quântico, para onde os excitons podem relaxar antes de sua recombinação.

A existência dos estados de menor energia, criados pela variação do potencial de confinamento, gera uma cauda exponencial na densidade de estados bidimensional do poço quântico, como esquematizado na Figura 6-(a). A recombinação radiativa dos portadores relaxados para estes estados forma uma cauda exponencial no lado de menor energia da curva de PL (Figura 6-(b)), que será tanto mais proeminente quanto maior for a magnitude das flutuações do potencial.

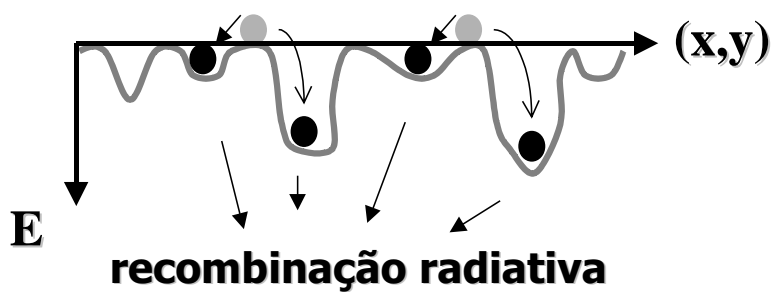

Figura 5. Potencial aleatório ao longo do plano (x,y) do poço quântico, formado pelas imperfeições estruturais. Os círculos claros representam portadores de carga fotogerados confinados no poço, e os círculos escuros representam excitons relaxados para os estados de menor energia criados pelas flutuações do potencial.

Trabalhos experimentais recentes, baseados em medidas que utilizam um feixe de excitação muito concentrado (diâmetro em torno de $10 \mu \mathrm{m}$ ) mostram que o lado de menor energia das curvas de PL é na verdade o envoltório de muitas linhas muito finas, que têm sua origem na recombinação de excitons relaxados para diferentes mínimos do potencial de confinamento (JAHN et al., 1996).

A difusão química de elementos na interface é um problema que ocorre devido a possíveis reações químicas entre os materiais na vizinhança da interface da heterojunção. Essas reações advêm dos procedimentos que são realizados durante a preparação da amostra, quando é feita a troca entre as fontes dos elementos que formam os diferentes compostos na heteroestrutura. Um exemplo é o sistema $\mathrm{Ga}_{0,52} \mathrm{In}_{0,48} \mathrm{P} / \mathrm{GaAs}$ (nesta composição da liga há casamento do parâmetro de rede com o GaAs). $\mathrm{Na}$ interface entre o GaInP e o GaAs, ocorrem dois processos: a interrupção das fontes que fornecem os elementos In e P, e a abertura da fonte do elemento As. Na interface inversa, a fonte de As é interrompida e as dos elementos In e P voltam a estar ativas. É possível que ocorra a incorporação de átomos residuais de In e $\mathrm{P}$ nas primeiras camadas crescidas de GaAs, assim como de As nas primeiras camadas crescidas de GaInP. Outro problema é a forte tendência à segregação do elemento In na liga GaInP (MESRINE et al., 1997), que produz uma frente de crescimento sempre rica em In, o que pode causar, por um lado, uma incorporação indesejada de In nas camadas de GaAs subseqüentes, e por outro, camadas iniciais de $\mathrm{Ga}_{\mathrm{x}} \mathrm{In}_{1-\mathrm{x}} \mathrm{P}$ pobres em In $(\mathrm{x}>0,52)$. Este fenômeno também foi verificado em sistemas InGaAs/GaAs (MURAKI et al., 1992).

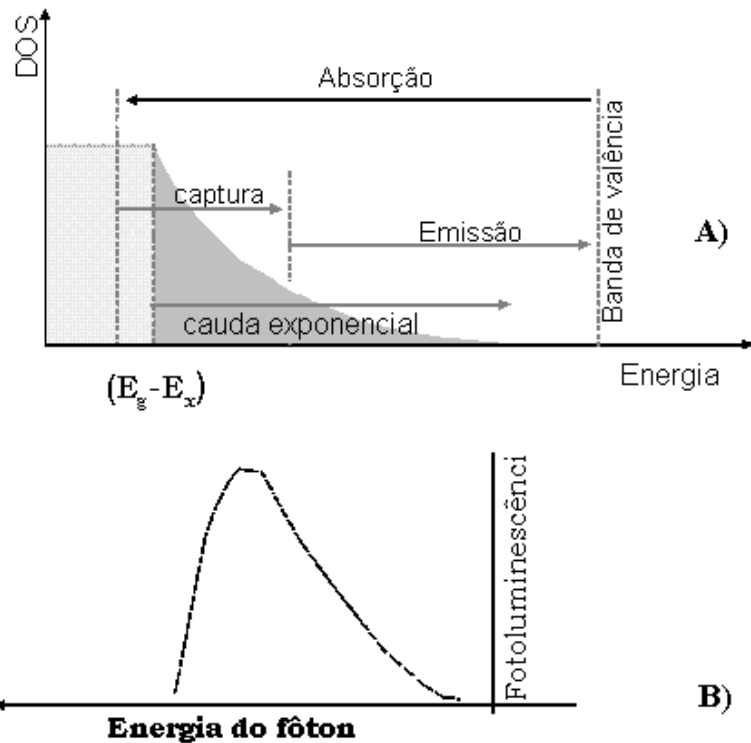

Figura 6. (a) Esquema da densidade de estados do poço quântico. Poço quântico com uma cauda exponencial, que reflete os estados de menor energia gerados pelas flutuações do potencial ao longo do plano do poço. $\mathrm{E}_{\mathrm{g}}-$ $\mathrm{E}_{\mathrm{x}}$ é a energia de recombinação do exciton livre, onde $\mathrm{E}_{\mathrm{x}}$ é a energia de ligação excitônica. (b) Forma da linha de PL, que se torna assimétrica no lado de menor energia dos fótons, devido às recombinações provenientes dos estados da cauda exponencial. 
Estes fenômenos combinados resultam na formação de compostos indesejados na região das interfaces, cuja estequiometria é diferente daquela dos materiais constituintes previstos para a formação da heteroestrutura, e que perturbam o potencial do poço (que seria perfeitamente retangular se as interfaces fossem composicionalmente abruptas). Essa deformação no potencial pode causar uma alteração significativa na posição em energia dos níveis, provocando um deslocamento na energia de emissão do poço que pode ser prejudicial para o desempenho de um dispositivo óptico. É portanto fundamental o entendimento e o controle do processo de formação das interfaces durante o crescimento das heteroestruturas, de maneira que o perfil de potencial real seja o mais próximo possível do perfil projetado.

\section{Detalhes Experimentais}

Nesta seção, será apresentada a montagem experimental da técnica de PL, além da descrição das amostras que foram utilizadas neste trabalho. Um esquema do aparato experimental utilizado para medidas de PL é mostrado na Figura 7.

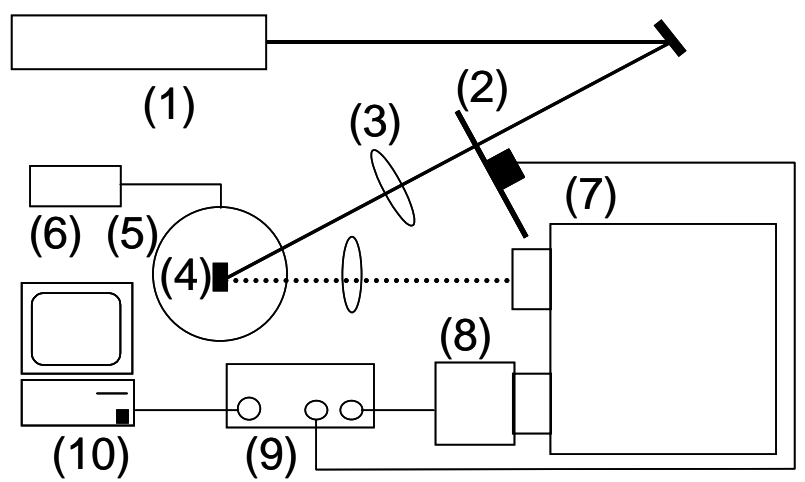

Figura 7. Esquema da montagem experimental utilizada para medidas de PL.

(1) Laser; (2) "chopper"; (3) lente convergente; (4) amostra; (5) criostato; (6) controlador de temperatura; (7) monocromador; (8) fotodetetor (ou fotomultiplicadora); (9) "lock-in"; (10) microcomputador com sistema de aquisição de dados .

O feixe de um LASER (geralmente de Argônio) é desviado por espelhos, passa por um recortador de luz (“chopper") e é focalizado sobre a superfície da amostra semicondutora (o "chopper" fornece uma referência para o sistema de detecção, como apontado a seguir). A amostra fica acomodada dentro de um criostato - do tipo fluxo contínuo ou de imersão de $\mathrm{He}$ - que permite baixar a temperatura até valores em torno de $10 \mathrm{~K}$. Um controlador pode ser utilizado para manter a temperatura a que a amostra está submetida. A luminescência da amostra (linha pontilhada na Figura 7) é coletada por uma lente e focalizada na fenda de entrada de um monocromador. O sinal é detectado por um fotodetetor (ou fotomultiplicadora) instalado na fenda de saída do monocromador. Este sinal elétrico é então enviado a um amplificador síncrono "lock-in" sintonizado na frequiência do "chopper", que o amplifica e o envia ao sistema de aquisição de dados. O sistema também coordena o movimento da rede de difração do monocromador, de modo que o resultado final é um gráfico de intensidade de luminescência em função do comprimento de onda $\lambda$. A conversão de $\lambda$ em energia é feita segundo a relação $E(\mathrm{eV})=12398.56 / \lambda(\AA)$.

$\mathrm{Na}$ Tabela 2, estão listadas as características principais das amostras estudadas, contendo os materiais que compõem o poço e as barreiras, respectivamente, assim como a espessura do poço em cada uma delas. As amostras foram separadas em três conjuntos, com a intenção de enfocar separadamente cada um dos diferentes aspectos das imperfeições estruturais discutidos na seção anterior.

Tabela 2. Características principais das amostras utilizadas neste trabalho.

\begin{tabular}{cccc}
\hline Amostra & Material do poço & Material das barreiras & $\mathrm{L}_{\mathrm{QW}}(\AA)$ \\
\hline I-A & $\mathrm{GaAs} \mathrm{A}$ & $\mathrm{Al}_{0.17} \mathrm{Ga}_{0.83} \mathrm{As}$ & 100 \\
I-B & $\mathrm{GaAs}_{0.8} \mathrm{Sb}_{0.2}$ & $\mathrm{Al}_{0.17} \mathrm{Ga}_{0.83} \mathrm{As}$ & 100 \\
I-C & $\mathrm{GaAs}_{0.843} \mathrm{Sb}_{0.15} \mathrm{~N}_{0.007}$ & $\mathrm{GaAs}$ & 150 \\
II-A & $\mathrm{GaAs}$ & $\mathrm{Ga}_{0.52} \mathrm{In}_{0.48} \mathrm{P}$ & 50 \\
II-B & $\mathrm{GaAs}$ & $\mathrm{Ga}_{0.52} \mathrm{In}_{0.48} \mathrm{P}$ & 50 \\
III-A & $\mathrm{GaAs}$ & $\mathrm{Ga}_{0.52} \mathrm{In}_{0.48} \mathrm{P}$ & 80 \\
III-C & $\mathrm{GaAs}$ & $\mathrm{Ga}_{0.52} \mathrm{In}_{0.48} \mathrm{P}$ & 30 \\
\hline
\end{tabular}

A segunda coluna diz respeito ao material que forma o poço quântico, e a terceira coluna se refere ao material das barreiras. A espessura da camada do poço está indicada na quarta coluna. 
No conjunto I, o material que forma o poço é um binário (GaAs) na amostra I-A, uma liga ternária (GaAsSb) na amostra I-B, e uma liga quaternária (GaAsSbN) na amostra I-C. O aumento da complexidade do material componente do poço aumenta a possibilidade de ocorrência de flutuações localizadas de sua composição química. Espera-se, assim, que os efeitos dessas flutuações sejam observados nos espectros de PL, e que se tenha uma idéia de sua magnitude através da comparação dos espectros das diferentes amostras.

No conjunto II, os materiais do poço (GaAs) e da barreira (GaInP) são os mesmos nas duas amostras, assim como suas espessuras nominais. Porém, foram seguidos diferentes procedimentos de crescimento (relatados mais adiante) durante a formação das interfaces, o que, devido aos problemas da mistura As/P e de segregação de In, pode levar à formação de compostos indesejados na interface cuja estequiometria seja consideravelmente diferente em cada caso. Tais compostos interfaciais podem afetar o perfil de potencial do poço e alterar a posição em energia dos níveis quantizados, de acordo com o discutido na Seção II-C. Com o emprego da técnica de PL, deseja-se averiguar se tais efeitos ocorreram nestas amostras, e se é possível inferir algo sobre a natureza química dos compostos interfaciais.

Por fim, o conjunto III é composto de duas amostras de poços quânticos de $\mathrm{GaInP} / \mathrm{GaAs}$ obtidas sob as mesmas condições de crescimento, com poços de diferentes espessuras: na amostra III-A a espessura do poço é de $80 \AA$ e na amostra III-B a espessura é de $30 \AA$. Mantendo as mesmas condições de crescimento, espera-se produzir interfaces estruturalmente semelhantes. No entanto, as rugosidades de interface devem se tornar mais significativas no poço de menor espessura, e esperase que isso seja refletido nos espectros de PL.

\section{Resultados e Discussão}

A Figura 8 traz os espectros de PL feita a $10 \mathrm{~K}$ das três amostras do conjunto I. Nos gráficos 8-(a), (b) e (c), são comparadas as curvas de emissão de heteroestruturas cujo poço é constituído de um material binário, ternário e quaternário, respectivamente. Nos gráficos mostrados, a origem do eixo-x coincide com a energia do máximo de intensidade de cada curva.

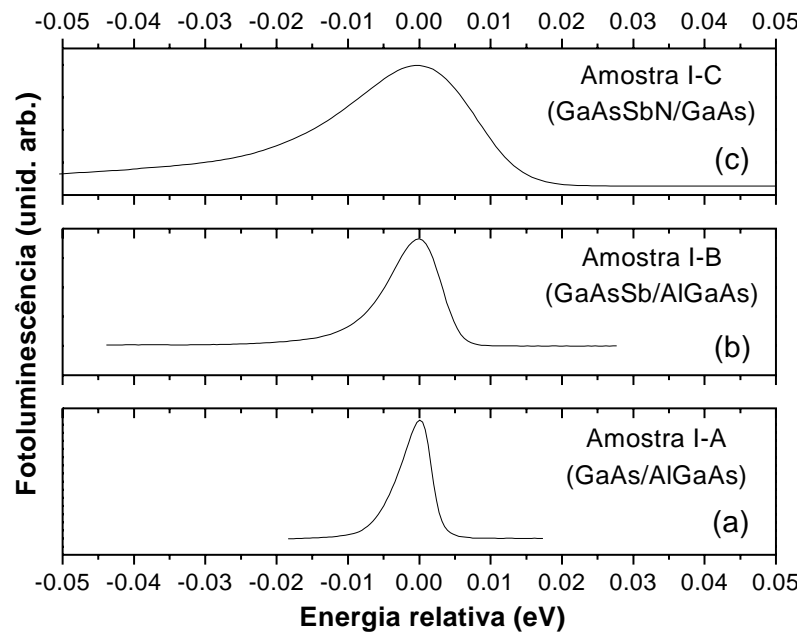

Figura 8. Espectros de PL das amostras I-A (a), I-B (b) e I-C (c). A referência para o eixo-x é a energia de máxima intensidade de PL de cada espectro. As medidas foram realizadas à temperatura de $10 \mathrm{~K}$.

Dois aspectos ficam evidentes pela comparação entre os espectros: primeiramente, observa-se um alargamento da curva à medida que mais elementos estão sendo empregados para compor o material do poço (no sentido de (a) para (c)); ao mesmo tempo, também no mesmo sentido, verifica-se uma crescente assimetria da curva para o lado de energia relativa negativa (lado de menor energia).

A desordem composicional no poço aumenta sucessivamente do material binário para o material ternário, e deste para o quaternário. Tal desordem gera as flutuações do potencial de confinamento, com o surgimento da cauda exponencial na densidade de estados do poço. $\mathrm{O}$ incremento na desordem aumenta a magnitude das flutuações, causando o alargamento da curva de PL. A recombinação radiativa a partir dos estados da cauda exponencial provoca a assimetria na curva de PL, de acordo como o previsto na Figura 6. As possíveis flutuações de composição no material das barreiras têm um efeito menor sobre 
os espectros de PL (BAJAJ, 2001), e podem ser desprezadas neste caso. A inclusão de Nitrogênio aumenta consideravelmente a desordem composicional em sistemas III- $\mathrm{V}_{1-\mathrm{x}} \mathrm{N}_{\mathrm{x}}$ (POLIMENI et al., 2000). A Figura 8 demonstra com muita clareza a maneira como os efeitos das imperfeições estruturais dos materiais componentes do poço são retratados nos espectros de PL.

Os resultados das medidas de PL sobre as amostras do conjunto II são apresentados na Figura 9. Na Figura 9-(a) está indicado o procedimento adotado em cada crescimento, onde as linhas verticais separam diferentes eventos do procedimento, e as linhas horizontais indicam a fonte do elemento (In, $\mathrm{Ga}$, As ou P) que está em atividade. Os números indicam o intervalo de tempo dos eventos relevantes para nossa análise.

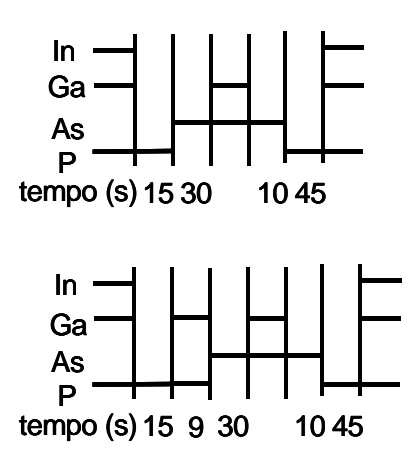

(a)

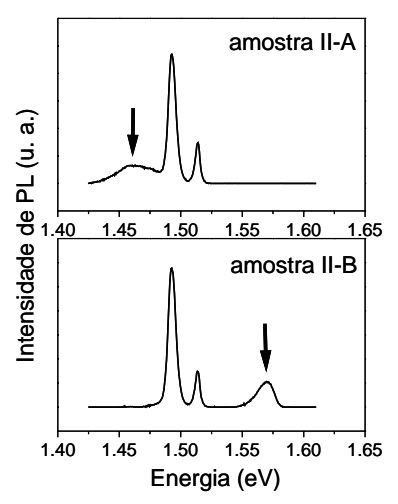

(b)
Figura 9. (a) Esquema dos procedimentos adotados nos crescimentos das amostras II-A e II-B; (b) Espectros de $\mathrm{PL}$ das respectivas amostras, tomados a $2 \mathrm{~K}$.

A segunda interface (GaAs-GaInP) é formada seguindo os mesmos procedimentos nas duas amostras. Este procedimento envolve a estabilização da superfície de GaAs durante 10 segundos com fluxo de As, e, posteriormente, uma exposição desta superfície ao fluxo de P durante 45 segundos. Desse modo, as variações se resumem nos procedimentos adotados para a primeira interface, a qual, seguindo relatos de diversos trabalhos (BEGOTTI et al., 2004), é a interface mais susceptível aos efeitos de difusão composicional no sistema GaInP/GaAs. Na amostra
II-A, após a estabilização com fluxo de P por 15 segundos, a superfície do GaInP ficou exposta ao As durante 30 segundos. Para a amostra II-B, sobre a superfície estabilizada do GaInP, foi depositada uma fina camada de $\mathrm{GaP}$ durante 9 segundos (o que resultou numa espessura de 3 monocamadas, segundo a taxa de crescimento utilizada) e, então, essa superfície ficou exposta ao As igualmente durante 30 segundos.

Os espectros de PL dessas amostras, tomados a $2 \mathrm{~K}$ sob mesmas condições experimentais, são mostrados na Figura 9-(b). São observados três picos, dois deles situados em energias idênticas nos dois gráficos (em torno de 1,5 eV). Eles correspondem às recombinações radiativas que ocorrem na camada "buffer" de GaAs (GRILLI et al., 1992). O outro sinal de PL vem da recombinação óptica envolvendo portadores confinados nos poços quânticos. Na amostra II-A esta emissão se dá com um valor de energia abaixo do valor do sinal proveniente da camada "buffer" de GaAs. Este é um resultado surpreendente, uma vez que o confinamento produz uma renormalização do "gap" (de acordo com o discutido na Secção II), e a emissão envolvendo os níveis fundamentais para elétron e para buraco no poço quântico deveria ocorrer com uma energia acima do "gap" do material que constitui o poço (no caso, o GaAs). A emissão relativa ao poço quântico na amostra II-B tem seu máximo em torno de 1,57 $\mathrm{eV}$, valor próximo do obtido através de previsões teóricas considerando um poço quântico ideal.

A emissão anômala (abaixo do "gap" do GaAs) pode ser explicada considerando a possibilidade que compostos indesejados tenham se formado na primeira interface da amostra II-A. A exposição direta da superfície do GaInP ao fluxo de As por um intervalo de tempo relativamente longo (30 segundos) pode gerar compostos quaternários do tipo GaInAs $\mathrm{P}_{1-\mathrm{y}}$ devido a uma mistura entre os elementos Pe As, cuja composição y, que pode variar sensivelmente ao longo da direção de crescimento, é função direta do tempo de exposição ao fluxo de As. Tais compostos podem ter "gap" inferior ao do GaAs, 
dependendo da composição relativa dos materiais V (valor de y). Sob sua influência, o perfil de potencial do poço pode ser seriamente deformado. Isso faz com que seus níveis fundamentais (para elétron e para buraco) surjam em valores de energia bem menores que para o caso do poço ideal, e sua separação pode até mesmo resultar numa energia menor que o "gap" do GaAs. De fato, cálculos dos níveis de energia para poços quânticos com estes compostos de baixo valor de "gap" inseridos nas interfaces dão conta de uma redução na energia de emissão de mais de $100 \mathrm{meV}$ (LAURETO, 2002). Imagens de Microscopia Eletrônica de Transmissão da região das interfaces na amostra II-A sugerem a presença de compostos $\mathrm{GaInAs}_{\mathrm{y}} \mathrm{P}_{1-\mathrm{y}}$ com altos valores de y (MARTINS et al., 2004).

Para o estudo do papel das rugosidades de interface na forma de linha da PL, serão utilizadas as amostras do conjunto III, consistindo de poços quânticos de GaInP/GaAs de diferentes espessuras (conforme Tabela II). Nestes crescimentos foram adotados procedimentos de maneira a minimizar a difusão composicional na região das interfaces (LAURETO et al., 2002).

Os espectros de PL realizada a $2 \mathrm{~K}$, utilizando baixíssimo nível de excitação, são apresentados na Figura 10. As emissões abaixo de 1,525 eV vêm da camada "buffer" e do substrato de GaAs, e não são de interesse para esta análise. As larguras a meia altura da emissão dos poços são, respectivamente, de 3,6 meV e 8,4 meV para as amostras III-A $\left(\mathrm{L}_{\mathrm{QW}}=80 \AA\right)$ e III-B $\left(\mathrm{L}_{\mathrm{QW}}=30 \AA\right)$, e isso indica que a influência das imperfeições de interface é mais forte na amostra com poço de menor espessura.

Levando em conta a aproximação do poço de potencial infinito, onde a energia do nível quântico é proporcional ao inverso do quadrado da espessura do poço $\left(E \propto L_{Q W}{ }^{-2}\right)$, pode-se mostrar que um maior valor da razão $\Delta \mathrm{L}_{\mathrm{QW}} / \mathrm{L}_{\mathrm{QW}}$ (onde $\Delta \mathrm{L}_{\mathrm{QW}}$ é a variação na espessura do poço causada pela rugosidade de interface) induz uma maior variação relativa na energia do nível. Este argumento, apesar de simplificado pela suposição do potencial infinito, pode ser aplicado para explicar o aumento da largura da PL com o decréscimo de $\mathrm{L}_{\mathrm{QW}}$, no caso de $\Delta \mathrm{L}_{\mathrm{QW}}$ permanecer constante. Pode-se concluir portanto que, mesmo que se mantenha o padrão de rugosidade nas interfaces, a influência deste padrão sobre os espectros ópticos será tanto maior quanto menor for a largura do poço quântico analisado.

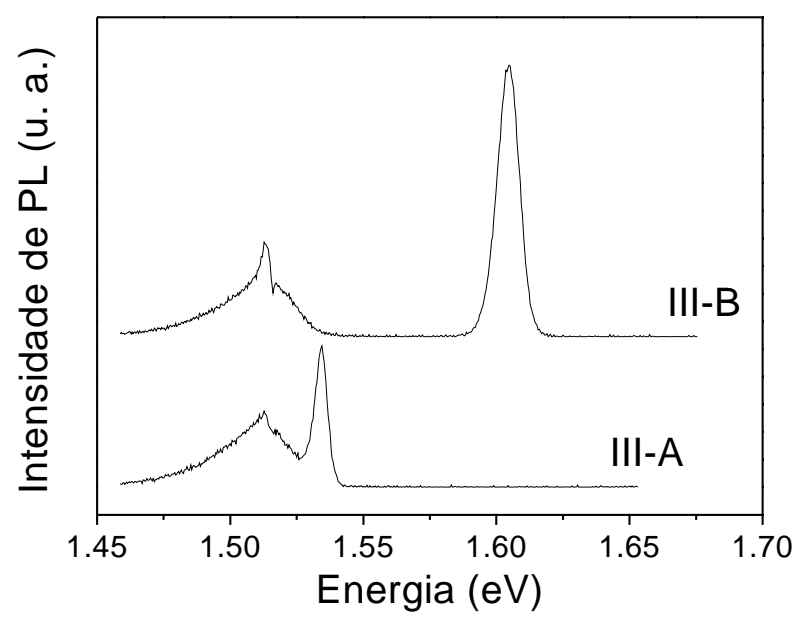

Figura 10. Espectros de PL a $2 \mathrm{~K}$ das amostras do conjunto III. A emissão dos poços ocorre em 1,533 eV e 1,603 eV para as amostras III-A e III-B, respectivamente. A emissão abaixo de 1,525 eV tem sua origem na camada "buffer" e no substrato de GaAs.

Em seguida, foram realizadas medidas de PL em função da temperatura. Alguns espectros estão incluídos na Figura 11-(a) para a amostra III-A, e na Figura 11-(b) para a amostra III-B. Estas medidas foram realizadas mantendo fixas todas as condições experimentais, variando somente a temperatura a que a amostra está submetida. 


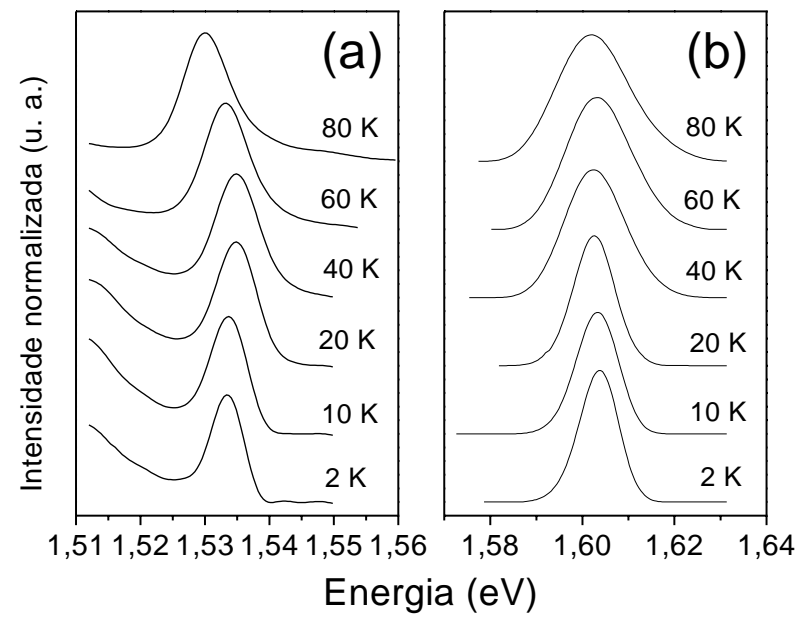

Figura 11. Espectros de PL (com intensidades normalizadas) das amostras III-A (a) e III-B (b), respectivamente, para diferentes temperaturas da amostra.

Na Figura 12, é mostrada a variação da posição em energia dos picos das curvas de PL em função da temperatura para as amostras III-A e III-B. Podese observar que as curvas experimentais apresentam comportamentos bastante distintos. Para a amostra III-A (triângulos), a curva inicialmente se desloca para maiores energias e depois decresce monotonicamente a partir de $40 \mathrm{~K}$. Para a amostra III-B (círculos), a curva se desloca primeiramente para menores energias, depois para maiores energias, e volta a decrescer depois de $70 \mathrm{~K}$. Tal comportamento difere completamente do verificado em amostras de GaAs (material volumétrico) na região de temperatura entre 0 e $80 \mathrm{~K}$, o qual, para temperaturas entre 0 e $40 \mathrm{~K}$, apresenta-se praticamente constante, e desloca-se monotonicamente para menores energias a partir de aproximadamente $50 \mathrm{~K}$ (GRILLI, 1992).

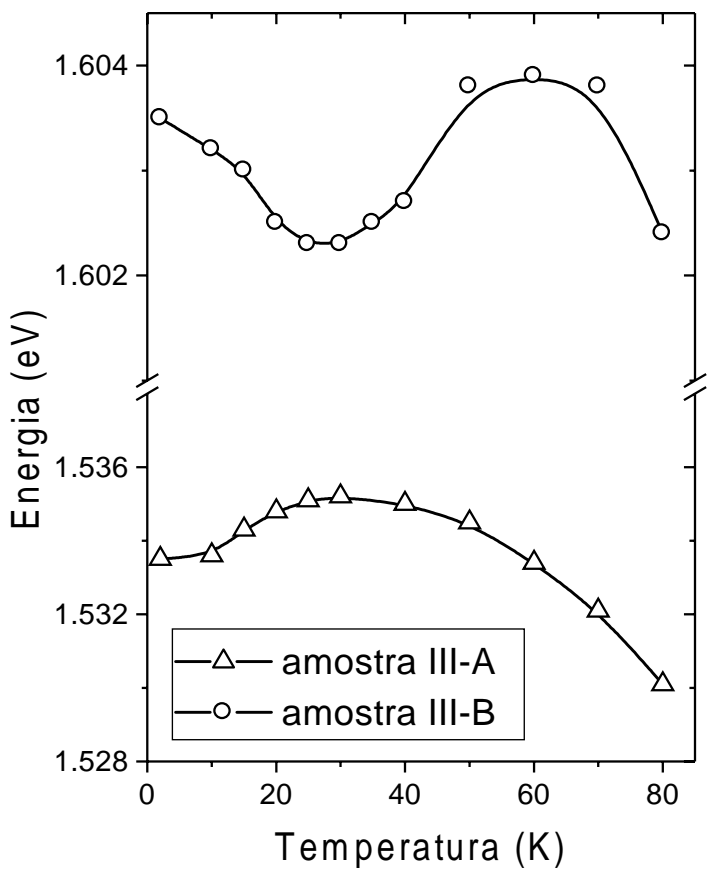

Figura 12. Variação da energia do pico de PL em função da temperatura para os poços quânticos de GaInP/GaAs com $\mathrm{L}_{\mathrm{QW}}=80 \AA$ (amostra III-A) e $\mathrm{L}_{\mathrm{QW}}=30 \AA$ (amostra III-B). A linha contínua é somente um guia para os olhos.

Para explicar essa variação do pico de PL com a temperatura, deve-se levar em conta a interação dos portadores fotogerados com as flutuações aleatórias do potencial de confinamento causadas pelas rugosidades nas interfaces dos poços quânticos, cujo esquema foi mostrado na Figura 5. Essas flutuações geram mínimos no potencial e sua profundidade depende das dimensões das rugosidades localizadas ao longo do plano do poço. Em uma medida de PL, os portadores de carga gerados pela excitação óptica tendem a relaxar para os estados de mais baixa energia antes da recombinação. Diante da configuração aleatória do potencial, os excitons formados poderão relaxar ou para mínimos locais, ou para o mínimo absoluto. O processo seguinte de termalização, onde os portadores relaxam para os estados de menor energia possível, é dependente da temperatura. A dinâmica deste processo é determinada pela relação entre a energia térmica kT (onde k é a constante de Boltzmann) e a profundidade do mínimo do potencial. 
Com o acréscimo da temperatura (incremento em kT), o exciton em um mínimo local do potencial poderá saltar para outro mínimo mais profundo (mínimo absoluto), ou escapar do mínimo de potencial e eventuamente recombinar-se de um nível energeticamente mais alto. A recombinação a partir dos níveis mais altos faz com que a curva de PL sofra um deslocamento para maiores energias, e a relaxação para mínimos mais profundos causa um deslocamento da PL para menores energias. Esse último efeito não é observado na amostra III-A, mas é evidente em III-B. De fato, como foi discutido acima, a magnitude das imperfeições de interface é realçada no poço mais estreito, gerando uma maior magnitude relativa das flutuações do potencial. Com maior incremento de temperatura, a energia térmica fornecida acaba sendo suficiente para que o exciton livre-se da influência até mesmo dos mínimos de potencial mais profundos, e o máximo da curva de PL desloca-se novamente para maiores energias. Dessa maneira, fica evidente que a variação na magnitude relativa das imperfeições de interface afeta também o processo de emissão espontânea dos poços, o qual dependerá da relação entre a profundidade dos mínimos do potencial e a energia térmica kT. O deslocamento da PL para menores energias em temperaturas mais altas $(\mathrm{T}>70 \mathrm{~K})$ segue o comportamento esperado de variação do "gap", governado principalmente pela interação elétron (exciton)-fônon (VIÑA; LOGOTHETIDIS; CARDONA, 1984; LAUTENSCHLAGER et al., 1987; LOURENÇO et al., 2004).

\section{Conclusões}

Imperfeições estruturais exercem forte influência sobre as propriedades ópticas de heteroestruturas semicondutoras que formam a base de diversos dispositivos optoeletrônicos e fotônicos atuais. Um claro entendimento da origem destas imperfeições é essencial para seu controle e minimização dos seus efeitos.

Neste trabalho, foram discutidos os aspectos gerais de imperfeições estruturais relacionadas às interfaces e à formação de compostos semicondutores envolvendo a mistura de três ou mais elementos químicos. Por meio da análise de diferentes sistemas semicondutores formando poços quânticos, foi possível verificar o alto grau de sensibilidade dos espectros de PL aos efeitos dessas imperfeições. Foram investigados três conjuntos de amostras. No conjunto I, com o aumento da complexidade do material que constitui o poço (passando de um binário para uma liga ternária e depois para uma liga quaternária), verificou-se um alargamento da curva de PL e o surgimento de uma cauda exponencial no lado de menor energia do espectro, decorrentes do aumento da desordem composicional nos poços. No conjunto II, foi observada a influência de compostos estequiometricamente indesejados, formados nas interfaces de poços quânticos de GaAs/GaInP, sobre a posição em energia do pico de emissão do poço. $\mathrm{E}$ no conjunto III, contendo amostras de poços quânticos de GaAs/GaInP de espessuras diferentes, foram analisados os comportamentos dos picos de emissão em função da temperatura. Tais comportamentos, na região de baixas temperaturas, são regidos pela configuração e pela magnitude relativa das imperfeições de interface. Com base nos resultados apresentados neste trabalho, verifica-se que a técnica de PL constitui-se numa valiosa ferramenta de investigação dos problemas relacionados à dinâmica do crescimento de materiais semicondutores e do processo de formação das interfaces em heteroestruturas semicondutoras.

\section{Agradecimentos}

Os autores expressam seus agradecimentos a $\mathrm{J}$. Bettini e W. Carvalho Jr. pelo crescimento das amostras de GaAs/GaInP, a A. A. Quivy, pela amostra de GaAs/AlGaAs, e a J. C. Harmand, pelas amostras de GaAsSb/AlGaAs e GaAsSbN/GaAs. Os autores agradecem o apoio financeiro da Fundação Banco do Brasil, Fundação Araucária, CNPq e CAPES. 


\section{Referências}

ANDREANI, L. C.; PASQUARELLO, A. Accurate theory of excitons in GaAs- $\mathrm{Ga}_{1-\mathrm{x}} \mathrm{Al}_{\mathrm{x}}$ As quantum wells. Physical Review B, New York, v.42, n.14, p.8928-8938, nov. 1990.

ALESSI, M.G.; FRAGANO, F.; PATANÈ, A.; CAPIZZI, M.; RUNGE, E.; ZIMMERMANN, R. Competition between radiative decay and energy relaxation of carriers in disordered $\mathrm{In}_{\mathrm{x}} \mathrm{Ga}_{1-\mathrm{x}}$ As/GaAs quantum wells. Physical Review B, New York, v.61, n.16, p.10985-10993, apr. 2000.

AKIYAMA, H.; YOSHITA, M.; PFEIFFER, L.N.; WEST, K.W.; PINCZUK, A. One-dimensional continuum and exciton states in quantum wires. Applied Physics Letters, New York, v.82, n.3, p.379-381, 2003.

BAJAJ, K.K. Use of excitons in materials characterization of semiconductor system. Materials Science and Engineering, Lausanne, v.34, n.2, p.59-120, 2001.

BASTARD, G. Wave mechanis applied to semiconductor heterostructures. Les Ulis Cedex: Les éditions de Physique, 1986.

BEGOTTI, M.; LONGO, M.; MAGNANINI, R.; PARISINI, A.; TARRICONE, L.; BOCCHI, C.; GERMINI, F.; LAZZARINI, L.; NASI, L.; GEDDO, M. Effect of the growth sequence on the properties of $\mathrm{InGaP} / \mathrm{GaAs} / \mathrm{InGaP}$ quantum wells grown by LP-MOVPE from group-V metalorganic sources. Applied Surface Science, Amsterdam, v.222, n.1/4, p.423-431, 2004.

CHEN, T. H.; HUANG, Y. S.; LIN, D. Y.; TIONG, K. K. Temperature dependent photoreflectance and photoluminescence characterization of GaInNAs/GaAs single quantum well structures. Journal of Applied Physics, New York, v.96, n.11, p.6298-6305, 2004.

CHRISTEN, J.; BIMBERG, D. Line shapes of intersubband and excitonic recombination in quantum wells: influence of final-state interaction, statistical broadening, and momentum conservation. Physical Review B, New Yorkv. 42, n.11, p.7213-7219, 1990.

CINGOLANI, R.; PLOOG, K. Frequency and density dependent radiative recombination processes in III-V semiconductor quantum wells and superlattices. Advances in Physics, London, v.40, n.5, p.535-623, 1991.

DAVIES, J. H. The physics of low-dimensional semiconductors. Cambridge: Cambridge University Press, 1998.

ESAKI, L.; TSU, R. Superlattice and negative differential conductivity in semiconductors. IBM Journal of Research and Development, Armonk, v.14, n.1, p.6-65, 1970.
FERNANDEZ, J. R. L.; NORIEGA, O. C.; SOARES, J. A. N. T.; CERDEIRA, F.; MENESES, E. A.; LEITE, J. R.; AS, D. J.; SHIROKA, D.; LISCHKA, K. Near band-edge optical properties of cubic GaN. Solid State Communications, New York, v.125, n.3/4, p.205-208, 2003.

GRILLI, E.; GUZZI, M.; ZAMBONI, R.; PAVESI, L. High precision determination of the temperature dependence of the fundamental energy bap in gallium arsenide. Physical Review B, New York, v.45, n.4, p.1638-1644, 1992.

JAHN, U.; KWOK, S. H.; RAMNSTEINER, M.; HEY, R.; GRAHN, H. T. Exciton localization, photoluminescence spectra, and interface roughness in thin quantum wells. Physical Review B, New York, v.54, n.4, p.2733-2738, 1996.

LAURETO, E. Influência das interfaces sobre as propriedades óticas de poços quânticos de GaInP/GaAs. 2002. Tese (Doutorado em Física) - Universidade Estadual de Campinas, Campinas.

LAURETO, E.; MENESES, E. A.; CARVALHO JR., W.; BERNUSSI, A. A; RIBEIRO, E.; SILVA, E.C.F.; OLIVEIRA, J.B.B. Optical studies of the correlation between interface disorder and the photoluminescence line shape in GaAs/ GaInP quantum wells. Brazilian Journal of Physics, São Paulo, v.32, n.2, p.314-317, 2002.

LAUTENSCHLAGER, P.; GARRIGA, M.; LOGOTHETIDIS, S.; CARDONA, M. Interband critical points of GaAs and their temperature dependence. Physical Review B, New York, v.35, n.17, p.9174-9189, 1987.

LOURENÇO, S. A.; DIAS, I. F. L.; DUARTE, J. L.; LAURETO, E.; POÇAS, L. C.; FILHO, D. O. T., LEITE, J. R. Thermal expansion contribution to the temperature dependence of excitonic transitions in GaAs and AlGaAs. Brazilian Journal of Physics, São Paulo, v.34, n.2A, p.517525, 2004.

MARTINS, M. R.; OLIVEIRA, J. B. B.; TABATA, A.; LAURETO, E.; BETTINI, J.; MENESES, E. A.; CARVALHO, M. M. G. Optical and structural properties of GaAs/GaInP quantum wells grown by chemical beam epitaxy. Brazilian Journal of Physics, São Paulo, v.34, n.2B, p.620-622, 2004.

MESRINE, M.; MASSIES, J.; VANELLE, E.; GRANDJEAN, N.; DEPARIS, C. Photoluminescence energy and interface chemistry of GaInP/GaAs quantum wells. Applied Physics Letters, New York, v.71, n.24, p.3552-3554, 1997.

MURAKI, K.; FUKATSU, S.; SHIRAKI, Y.; ITO, R. Surface segragation on In atoms during molecular-beam epitaxy and its influence on the energy levels in InGaAs/GaAs quantum wells. Applied Physics Letters, New York, v.61, n.5, p.557, 1992 . 
OLIVEIRA, J. B. B.; MENESES, E. A.; DA SILVA, E. C. F. Magneto-optical studies of the correlation between interface microroughness parameters and the photoluminescence line shape in $\mathrm{GaAs} / \mathrm{Ga}_{0.7} \mathrm{Al}_{0.3} \mathrm{As}$ quantum wells. Physical Review B, New York, v.60, n.3, p.1519-1522, 1999.

POLIMENI, A.; CAPIZZI, M.; GEDDO, M.; FISCHER, M.; REINHARDT, M.; FORCHEL, A. Effect of temperature on the optical properties of $(\mathrm{InGa})(\mathrm{AsN}) / \mathrm{GaAs}$ single quantum wells. Applied Physics Letters, New York, v.77, n.18, p.2870-2872, 2000.

SHUR, M. Physics of semiconductor devices. London: Prentice-Hall International, 1990.

SINGH, J. Physics of semiconductors and their heterostructures. New York: McGraw-Hill Inc., 1993.

SINGH, J.; BAJAJ, K.K. Role of interface roughness and alloy disorder in photoluminescence in quantum-well structures. Journal of Applied Physics, New York, v.57, n.12, p.5433-5437, 1985.

VIÑA, L.; LOGOTHETIDIS, S.; CARDONA, M. Temperature dependence of the dieletric function of germanium. Physical Review B, New York, v.30, p.1979, 1984.

VURGAFTMAN, I.; MEYER, J. R.; RAM-MOHAN, I. R. Band-parameters for III-V compound semiconductors and their alloys. Journal of Applied Physics, New York,v.89, n.11,p.5815-5875, 2001.
WARE, M. E.; BRACKER, A. S.; STINAFF, E.; GAMMON, D.; GERSHONI, D.; KORENEO, V. L Polarization spectroscopy of positive and negative trions in an InAs quantum dot, Physica E, v.26, p.55, 2005.

WEISBUCH, C.; MILLER, R. C.; DINGLE, R.; GOSSARD, A. C.; WIEGMANN, W. Intrinsec recombination from quantum states in $\mathrm{GaAs}-\mathrm{Al}_{\mathrm{x}} \mathrm{Ga}_{1-\mathrm{x}}$ As multi-quantum well structures. Solid State Communications, New York, v.37, p.219, 1981.

XU, X; WILLIAMS, D.A.; CLEAVER, J.R.A. Splitting of excitons and biexcitons in coupled InAs quantum dots molecules, Applied Physics Letters, v. 86, p. 012103, 2005.

YOFFE, A. D. Semiconductor quantum dots and related systems: electronic, optical, luminescence and related properties of low dimensional systems, Advances in Physics, London, v.50, n.1, p.1-208, 2001.

YU, P.Y.; CARDONA, M. Fundamentals of semiconductors. Berlin: Springer-Verlag, 1996.

ZIMMERMANN, R.; RUNGE, E. Excitons in narrow quantum wells: disorder localization and luminescence kinetics. Physica Status Solidi A-Applied Research, Berlin, v.164, n.1, p.511-516, 1997. 